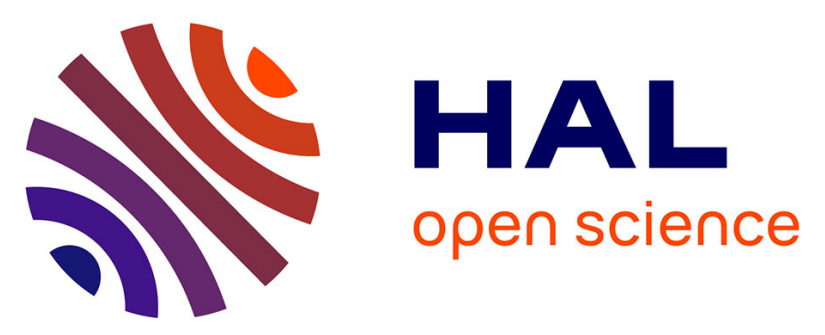

\title{
Influence of expressive versus mechanical musical performance on short-term memory for musical excerpts
} Barbara Tillmann, William Jay Dowling, Philippe Lalitte, Paul Molin, Katrin Schulze, Bénédicte Poulin-Charronat, Daniele Schon, Emmanuel Bigand

\section{- To cite this version:}

Barbara Tillmann, William Jay Dowling, Philippe Lalitte, Paul Molin, Katrin Schulze, et al.. Influence of expressive versus mechanical musical performance on short-term memory for musical excerpts. Music Perception, 2013, 13 (4), pp.419-425. 10.1525/mp.2013.30.4.419 . halshs-01188638

\section{HAL Id: halshs-01188638 \\ https://shs.hal.science/halshs-01188638}

Submitted on 10 Apr 2017

HAL is a multi-disciplinary open access archive for the deposit and dissemination of scientific research documents, whether they are published or not. The documents may come from teaching and research institutions in France or abroad, or from public or private research centers.
L'archive ouverte pluridisciplinaire HAL, est destinée au dépôt et à la diffusion de documents scientifiques de niveau recherche, publiés ou non, émanant des établissements d'enseignement et de recherche français ou étrangers, des laboratoires publics ou privés. 


\section{Influence of Expressive Versus Mechanical Musical Performance on Short-term Memory for Musical Excerpts}

Barbara Tillmann

Lyon Neuroscience Research Center, Lyon, France

W. JAY Dowling

University of Texas at Dallas

Philippe Lalitte \& Paul Molin

Université de Bourgogne, Dijon, France

Katrin Schulze

Lyon Neuroscience Research Center, Lyon, France \& UCL Institute of Child Health, London, UK

Bénédicte Poulin-Charronnat

Université de Bourgogne, Dijon, France

Daniele Schoen

Institut de Neurosciences des Systèmes, Marseille, France

Emmanuel Bigand

Université de Bourgogne, Dijon, France \& Institut

Universitaire de France, France

RECOGNITION MEMORY FOR DETAILS OF MUSICAL phrases (discrimination between targets and similar lures) improves for up to $15 \mathrm{~s}$ following the presentation of a target, during continuous listening to the ongoing piece. This is attributable to binding of stimulus features during that time interval. The ongoing-listening paradigm is an ecologically valid approach for investigating short-term memory, but previous studies made use of relatively mechanical MIDI-produced stimuli. The present study assessed whether expressive performances would modulate the previously reported finding. Given that expressive performances introduced slight differences between initially presented targets and their target-test items, expressive performance could make the task more difficult overall than did the previously used mechanical renderings. However, results revealed an even stronger improvement for the expressive pieces than for the mechanical pieces. The pattern of results was observed for participants varying in their level of musical experience, though the difference between expressive and mechanical conditions was more pronounced for the less-experienced participants. Overall, our study showed that the memory improvement phenomenon extends to more realistic musical material, which includes expressive timing characteristics of live performance.

Received: June 1, 2012, accepted August 6, 2012.

Key words: short-term memory, melodies, binding, expressivity, memory improvement

I N CONTRAST TO SHORT-TERM MEMORY FOR word lists or prose, which is subject to interference from similar materials (e.g., Tillmann \& Dowling, 2007), short-term memory for musical details improves over time during continuous listening to music. Dowling, Tillmann, and Ayers (2001) presented listeners with classical minuets in which an initial excerpt was tested later. The music continued for 4 to $15 \mathrm{~s}$ (as originally created by the composer) and recognition memory was tested with a repetition of the target (the test item, T), a similar lure (S-lure), or a different lure (D-lure). Short-term memory performance, especially participants' capacity to discriminate targets from similar lures (T/S discrimination), improved with increasing delay. This improvement effect has been observed for musician and nonmusician listeners, and with classical minuets and popular guitar music (Dowling, Tillmann, \& Magner, 2013). This musical ongoing-listening paradigm is an ecologically valid approach for investigating short-term memory: The music continues while listeners process and memorize the preceding musical information. The present study aimed to go further in this direction by applying this methodology to natural, expressive performances.

In previous studies, the observed improvement of $\mathrm{T} / \mathrm{S}$ discrimination over time has been attributed to the binding of separate features of the musical phrases (e.g., contour, scale) while the listener continues to listen. For example, in Beethoven's Minuet (Figure 1), the first phrase (in the key of $\mathrm{G}$ ) has a characteristic melodic

Music Perception, volume 30, issue 4, pp. 419-425, issn 0730-7829, electronic issn 1533-8312. (C) 2013 by the Regents of the university of California all RIGHTS RESERVED. PLEASE DIRECT ALL REQUESTS FOR PERMISSION TO PHOTOCOPY OR REPRODUCE ARTICLE CONTENT THROUGH THE UNIVERSITY OF CALIFORNIA PRESS'S RIGHTS AND PERMISSIONS WEBSITE, HTTP://WWW.UCPRESSJOURNALS.COM/REPRINTINFO.ASP. DOI: 10.1525/MP.2013.30.4.419 

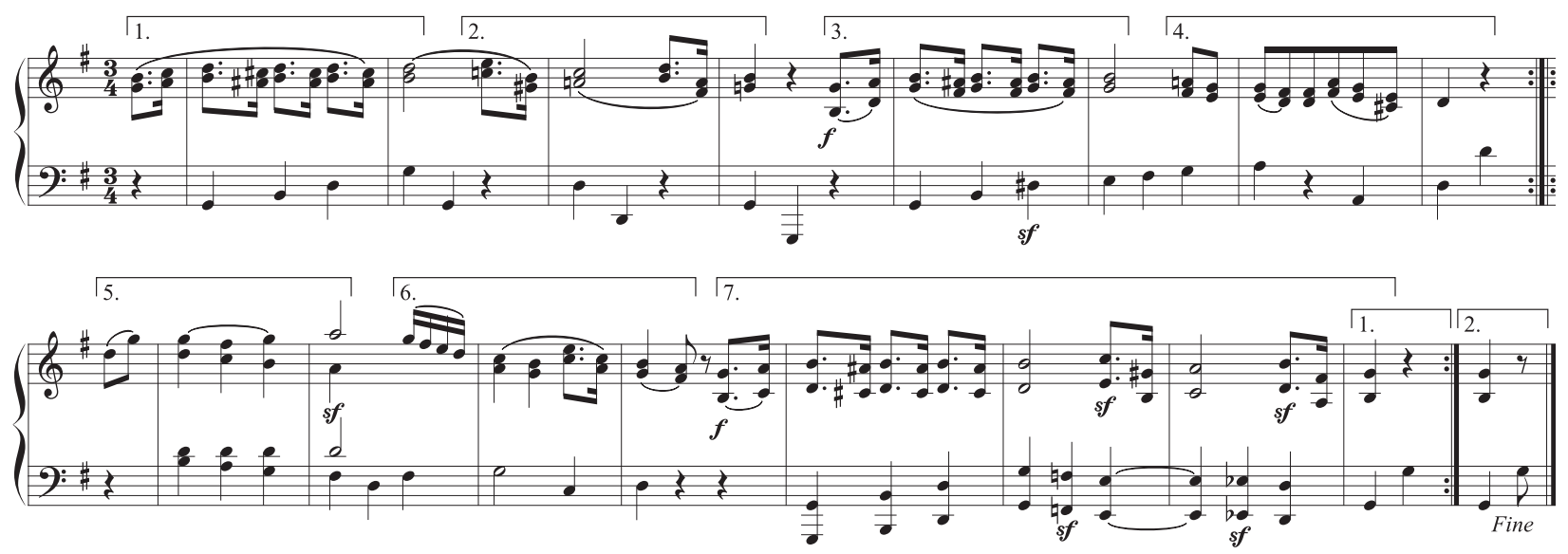

FIGURE 1. Beethoven's Minuet in G as stimulus example. The brackets indicate placement of possible target and test phrases. The :|| sign indicates an optional repeat of the material preceding this sign before continuing with the remaining part of the minuet (bracket 5). Bracket 1 indicates a test phrase that could be tested after a short delay at bracket 3 with an $\mathrm{S}$ lure. The phrase at bracket 2 could be tested after a long delay with a test item ( $\mathrm{T}$ ) comparison (an exact copy of the original phrase) by taking the repeat. Bracket 4 indicates a potential test with a $D$ lure after a short delay of bracket 2. The phrase at bracket 6 can test bracket 2 after a long delay with a D lure in case the repeat is not taken.

contour. The third phrase repeats those features, but with the melody shifted in pitch so that the contour connects to the scale at different places. This phrase could serve as a similar lure (S) for testing the first phrase after a 4-s delay. In that case, participants would base their judgments on individual features of the phrases, and as S lure and target phrase share features, participants tend to confuse the two (leading to high false-alarm rates for $\mathrm{S}$ lures). After $15 \mathrm{~s}$, the individual features have been bound together-the contour to the scale framework-and the confusion disappears. This feature-binding process closely parallels the operations leading to the formation of an "object file" in Treisman's Feature Integration theory (Treisman, 2006). The process appears to be slowed by the listener's attending to the ongoing music; if the delay interval is silent there is hardly any confusion and the performance level is higher starting at the short delay (Experiment 3, Dowling et al., 2001). This ongoing-listening paradigm thus provides an ideal way to study the binding process in musical memory.

Our experiments with minuets (Dowling et al., 2001; Dowling \& Tillmann, 2013) used MIDI edited versions to precisely control the timing. Even though the MIDIrenderings were not completely mechanical (they contained some expressive cues related to articulation, phrasing, and dynamics), a uniform tempo was imposed over the entire musical set and strict metrical structure was respected throughout. The tempo was selected to produce the appropriate delay between the introduction of a target item and its test, which led to relatively slow tempi for some of the minuets. For example, in Dowling et al. (2001, Experiment 1), the tempo of 72 beats/min produced delays of 5 and $15 \mathrm{~s}$, corresponding to two and six measures. Furthermore, each test item $\mathrm{T}$ was an exact repetition of the target item presented at the beginning of the minuet, inserted after a short or long delay.

The goal of our present study was to investigate the phenomenon of memory improvement over time during ongoing listening with more realistic musical material, which included expressive timing characteristics of live performances. Expressive performances allowed for dynamic and temporal changes, and the overall tempo of each minuet was chosen to get the most expressive and pleasant rendering. The expressive performances also included variations between targets and corresponding test items on tempo, tone duration, and exact metrical placement, as well as variations of loudness depending on the musical structure. This aspect of the expressive material, which is characteristic of real musical material, might thus make the short-term memory test more challenging for participants as comparisons had to be made on higher structural levels. However, expressive performances highlight underlying musical structures, which might enhance the involved memory and binding processes, thus leading to overall better performance. For example, Palmer (1996) found timing in a live performance to be correlated with structural harmonic tension in a piece defined in terms of Lerdahl and Jackendoff's (1983) theory, in the sense that the pianist tended to slow down at points of higher tension. Clarke 
and Windsor (2000) also found expressive timing to be effective in communicating structural differences. Although expressive and mechanical performances can lead to similar parsing of phrases (Palmer \& Krumhansl, 1987), and recent research has found the same electrophysiological markers for music-syntactic processing under both performance conditions, some evoked potentials were nevertheless shown to be enhanced for expressive performance (Koelsch \& Mulder, 2002; Koelsch, Kilches, Steinbeis, \& Schelinski, 2008). Furthermore, expressive performances have more power to evoke emotions than mechanical renderings (e.g., Chapin, Jantzen, Scott Kelso, Steinberg, \& Large, 2010; Viellard, Roy, \& Peretz, 2011). Our study compared memory performance for expressive piano performances and more mechanical renderings, which eliminated fluctuations in features related to tempo and duration fluctuations as well as use of the damper pedal, and which used exact repetitions as test items.

\section{Method}

\section{PARTICIPANTS}

Eighty-six undergraduates at the University of Texas at Dallas participated as part of their course requirements in psychology. Forty-four were categorized as moderately experienced and had at least three years of explicit music training (defined as lessons on an instrument or voice, or playing in an instrumental ensemble; $M=6.86 \mathrm{yr}, S D=$ 3.06 yr). Forty-two participants with less training were categorized as musically less-experienced. The factor version (expressive, mechanical) was used as a betweenparticipants factor, as repetition has been shown to change memory for musical pieces (Gardiner, Kaminska, Dixon \& Java, 1996; Peretz, Gaudreaux, \& Bonnel, 1998). Participants were distributed into four groups: 42 heard the expressive versions (20 less-experienced, 22 moderately experienced) and 44 heard the mechanical versions (22 less-experienced, 22 moderately experienced).

\section{MATERIAL}

Forty-eight minuets were selected from previously used stimuli (Dowling \& Tillmann, 2013; Dowling et al., 2001). The stimuli were drawn from classical minuets, waltzes, German dances, and Ländler written for piano between 1750 and 1828 by Haydn, Mozart, Beethoven, and Schubert. See http://www-crnl.univ-lyon1.fr/btsound.html for some sound examples.

One of the first two phrases of the piece (roughly two measures) was chosen as a target to be tested later. To allow presenting the music as originally composed, the pieces were selected so that the target phrase was neither repeated nor imitated in the continuation of the piece prior to the test. Test phrases occurred within the piece after a short (two measures) or long (six measures) delay. The test phrase was either a repetition of the target item (referred to as the test item T), or an imitation of the target that changed one or more features (such as pitch level), but left the melodic and rhythmic contour largely intact (S), or a new phrase not heard before in the piece (D).

The music was presented without any indication about where the target phrase was situated. Following the presentation of the target, the music continued just as written by the composer. The onset of the test was signaled by a soft, high-pitched tone (B two octaves above the treble staff) recorded with the same timbre as the minuet and that did not interfere with the music, occurring one-half beat before the test. The music stopped after presentation of the test phrase (T, S, or D) and participants were given $8 \mathrm{~s}$ to respond before the beginning of the next trial. On each trial a new piece appeared, of which participants heard the first 6 to 12 measures.

The experiment was run using MATLAB software, which randomized the order of presentation of the trials differently for each group of six participants (who were tested together in group sessions).

Expressive performances. The minuets were played by an expert pianist and recorded with an acoustic piano Yamaha Disklavier HQ100SX, an acoustic piano that provides for MIDI transcription, including use of the damper (sustain) pedal. The MIDI-files were edited using Cubase SX to correct errors of pitch and articulation. The MIDI-files were then transformed into audio files (.wav) using the Yamaha acoustic piano timbre. No constraints were given to the pianist, freely allowing for expressive dynamic and temporal changes. The pianist chose an overall tempo for each minuet to achieve the most expressive and pleasant performance. On average, the tempo of the minuets was $127 \mathrm{bpm}$ (ranging from 54 to 217, with $S D=34$ and a median of 131). Consequently, there was variation (but no overlap) between short and long delays. The beep indicating the test was presented after the following mean delays: $2.48 \mathrm{~s}(S D=0.87$, ranging from 1.53 to 5.53$)$ for short delays and $9.49 \mathrm{~s}(S D=2.37$, ranging from 6.85 to 14.28) for long delays (Table 1). Note that the target and test items were not identical renderings, and differed in expressive cues.

Mechanical versions. To construct the MIDI-files of the mechanical versions, the MIDI-files of the expressive versions were edited so that they respected the characteristics of the material in previous studies 
TABLE 1. Mean Delays in Seconds Between Targets and Test Items $(T, S, D)$ for the Short and Long Delay Conditions for the Three Types of Test.

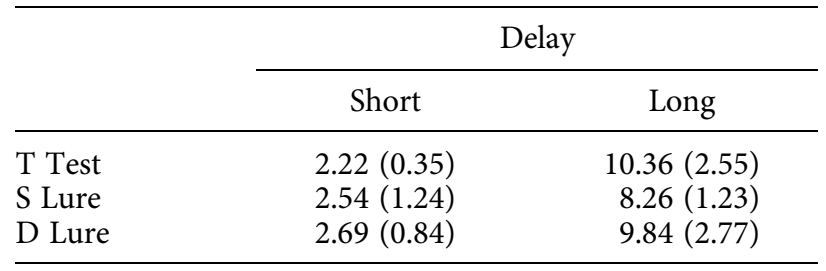

Note: Standard deviations are indicated in parentheses.

(Dowling \& Tillmann, 2013; Dowling et al., 2001), except for differences in tempo. Each mechanical version was matched in average tempo to the corresponding minuet's expressive version. Hence the principal differences between mechanical and expressive versions were: (1) the steady tempo that continued through the test item $\mathrm{T}$ in the mechanical version; and (2) the complete match between targets and $\mathrm{T}$ items in the mechanical version (thus contrasting with possible variations in tempo, loudness, balance, articulation, and phrasing of expressive version Ts). The mechanical versions were recorded on a Clavinova PF with attention to articulation and phrasing and balance among the parts. They were manipulated using CakeWalk software to ensure a steady tempo and precise alignment of notes on the beat and its subdivisions, and recorded as .wav files using a Yamaha acoustic grand piano timbre.

\section{PROCEDURE}

Participants were told that one of the first few phrases of each musical piece would serve as a target phrase to be tested later. They were informed that the target phrase, which they needed to remember, was not indicated by an explicit signal, but that there was a signal (i.e., a high-pitched tone, "ding") that indicated the start of the test phrase. Their task was to say whether the test phrase was an exact match to any of the phrases they had heard in that excerpt. We explained that the same melody could be shifted in pitch, and that would make it different, or that the same rhythm could be paired with a different melody, making it different, too. Participants were instructed to respond using a six-point confidence-level scale on which " 6 " = "very sure same," " $5 "$ " "sure same," "4" = "same," " 3 " = "different," " 2 " = "sure different," and " 1 " = "very sure different." We emphasized that the response scale was not intended as a rating of how different the target and test were, but as indicating how sure they were of their decision to have detected differences. Participants were given an explanation of the task with two examples of each trial type
(T, S, and D), illustrating the various kinds of difference that might occur between target and test. Participants also completed a brief questionnaire concerning musical experience.

\section{DATA ANALYSIS}

Responses to the three test item comparisons were reduced to two areas under the MOC (Memory Operating Curve), one assessing discrimination between $\mathrm{T}$ items and $\mathrm{S}$ lures, and the other discrimination between Ts and D lures. The six-point scale provided five criterion placements on the MOC with which to calculate the area. Area under the MOC provides an unbiased estimate of proportion correct where chance is .50 (Swets, 1973). The area score provides a better measure of performance than, for example, $d$ ', since it preserves more response information and is generally uncorrelated with measures of bias such as $c$ (unlike $d$ '; Dowling, Kwak, \& Andrews, 1995; Verde, Macmillan, \& Rotello, 2006). The 48 minuets covered the six conditions (two delays $\times$ three test phrase types) with eight trials in each condition. This meant that each area score was based on 16 data points: eight hit rates and eight false-alarm rates. We further report proportions of hits to Ts and false alarms to $S$ and $D$ lures.

\section{Results}

\section{AREA SCORES}

Area scores were subjected to a $2 \times 2 \times 2 \times 2$ repeatedmeasure ANOVA with Delay (short/long) and Item Comparison (T/S, T/D) as within-participant variables, and Version (expressive/mechanical) and Musical Experience (less-experienced/moderately experienced) as between-participants factors. Performance was overall slightly better for the moderately-experienced participants (.78) than for the less-experienced participants (.73), $F(1,82)=6.03, p<.05, M S E=491.32$. T/D discrimination (.82) was better than T/S discrimination (.70), $F(1,82)=143.25, p<.0001, M S E=66.41$. The interactions between Delay and Item comparison, $F(1,82)=92.50, p<.0001, M S E=47.20$, and between Delay and Version were significant, $F(1,82)=4.42, p<$ $.05, M S E=142.04$, but were further expressed in the expected three-way interaction of Delay $\times$ Version $\times$ Item Comparison (Figure 2), $F(1,82)=10.02, p<.01$, $M S E=47.20$. For $\mathrm{T} / \mathrm{S}$ discrimination, improvement over time was stronger for expressive versions, $t(82)$ $=4.53, p<.001$, than for mechanical versions, $t(82)$ $=1.26, p<.12$. For $\mathrm{T} / \mathrm{D}$ discrimination, the decrease of performance over time remained significant for expressive, $t(82)=2.39, p<.05$, and mechanical 


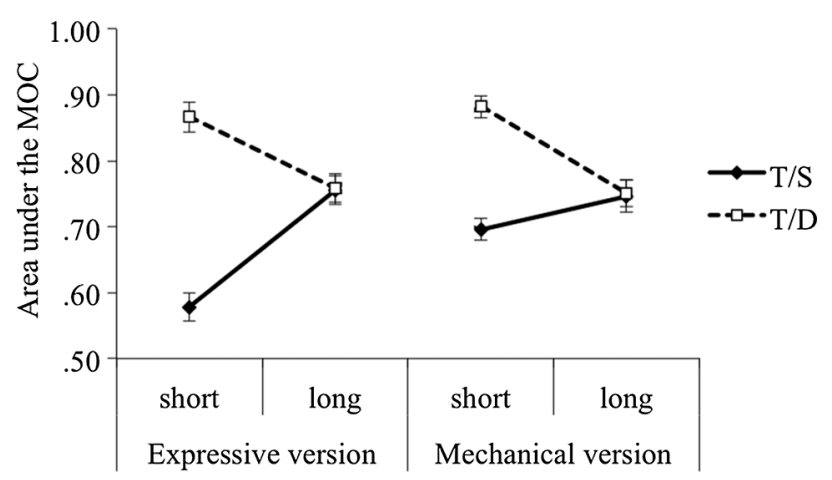

FIGURE 2. Percent area under the MOC presented as a function of version (expressive/ mechanical) and discrimination (T/S: discrimination of test items $(T)$ from similar lures (S); T/D: discrimination of test items $(T)$ from different lures $D)$. Error bars indicate between-participants standard errors.

versions, $t(82)=3.31, p<.01$. No other effects were significant. Note that the difference between expressive and mechanical versions for the T/S improvement was strongest for the less-experienced participants, but the three-way interaction was not significantly modulated by musical experience, $p=.10$.

PROPORTIONS OF HITS AND FALSE ALARMS

Proportions of hits and false alarms (Figure 3) were analyzed with a $2 \times 3 \times 2 \times 2$ ANOVA with Delay (short/ long) and Test item (T, S, D) as within-participant factors and Version (expressive/mechanical) and Musical
Experience (less experienced/moderately experienced) as between-participants factors. Here main effects involve a mixture of criterion shifts and differences in sensitivity, and so interactions were the main interest. A significant effect of Test item indicated that participants discriminated between target items and lures, $F(2,164)=311.52, M S E=.04, p<.0001$. The effect of Musical Experience was significant, $F(1,82)=4.48$, $M S E=.06, p<.05$, and interacted with Test item, $F(2,164)=6.94, M S E=.04, p<.01$, showing that the more experienced participants better discriminated Ts from lures. The effect of Delay was significant, $F(1,82)=259.41, M S E=.02, p<.0001$, and found expression in a two-way interaction with Test item, $F(2,164)=40.42, M S E=.02, p<.001$, a three-way interaction with Test item and Version, $F(2,164)=$ $6.72, M S E=.02, p<.01$, and a four-way interaction with Test item, Version, and Musical Experience, $F(2,164)=$ $6.03, M S E=.02, p<.01$. Going from the short delay to the long delay, hit rates decreased for $\mathrm{T}$ items and false alarms decreased for both S and D lures; the decrease of false alarms being stronger for $\mathrm{S}$ lures than for $\mathrm{D}$ lures. This decrease for $S$ lures was particularly strong for inexperienced listeners with the expressive versions (see Figure 3).

\section{Discussion}

Our experiment aimed at extending the previously observed memory improvement of fine details from

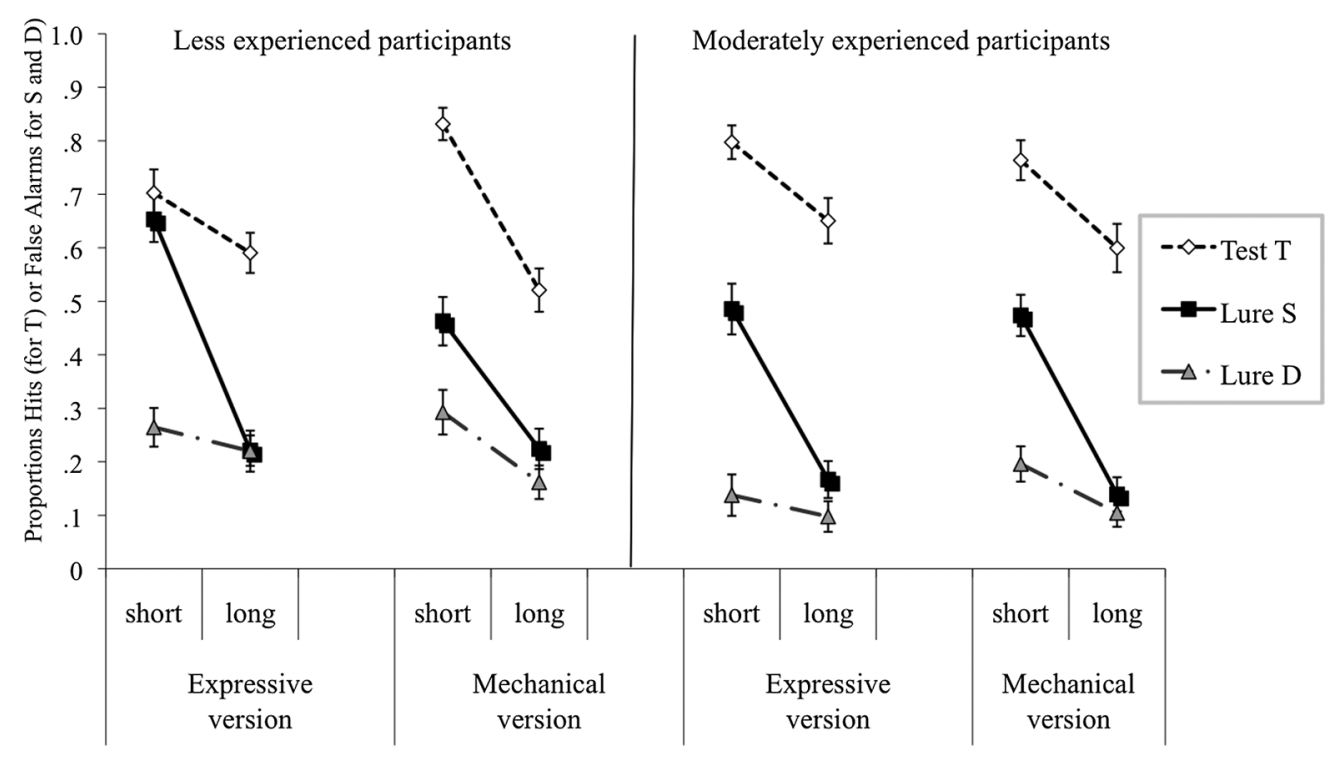

FIGURE 3. Proportions of hits (for test-items T) and false alarms (for similar lures $S$ and different lures D) presented as a function of version (expressive/mechanical) and test item (T), similar lures (S), and different lures (D) for less-experienced participants (left) and moderatelyexperienced participants (right). Error bars indicate between-participants standard errors. 
mechanical renderings to expressive performance. This allowed getting closer to the functioning of short-term memory in a more realistic listening situation, which combined continuous listening and expressive renderings: In the continuous listening situation, listeners did not know in advance which phrase would be tested later, and the intervening material was interfering material that is related to both the target and the test items. When using expressive renderings, as a consequence, the $\mathrm{T}$ item was not an exact copy of the original target item (presented earlier in the piece), but differed by changes introduced by the pianist in the course of playing the stimuli (i.e., timing, dynamics, and articulation). Three main findings were observed for memory of fine details (involving target-test items $\mathrm{T}$ and similar lures $S$ ):

First, with expressive performances, we replicated the improvement in T/S discrimination over the first $15 \mathrm{~s}$ following the presentation of a target phrase, similar to the results obtained with mechanical renderings (Dowling et al., 2001).

Second, participants were able to remember details of musical phrases over time, even though expressive versions could have made the task more difficult. Indeed, with the expressive versions, listener's "same" response no longer referred to an acoustically identical event, but rather to structurally the same musical event.

Third, data at the short delay revealed lower area scores for T/S discrimination with expressive performances than with mechanical versions. This lower performance was reflected in increased false alarm rates for $\mathrm{S}$ lures and lowered hit rates for $\mathrm{T}$ items in lessexperienced listeners. Due to the faster tempi of the expressive performances, the average "short delay" was $2.5 \mathrm{~s}$, and thus half the length of the short-delay condition in Dowling et al. (2001). With this short delay, an "echoic" trace of the target stimulus might have been available at the time of the test (e.g., Kaernbach et al., 2004). This echoic trace might provide an advantage for the mechanical condition, for which target and test items were identical renderings, but a disadvantage for the expressive condition as the slightly different $\mathrm{T}$ item and the somewhat different $S$ lure might become more easily confused with each other (note that T/S discrimination was significantly above chance level.) This interpretation is also supported by the observation that T/S discrimination for the mechanical material in the present experiment was better than in the previous study using 5 -s delays (area score .70 versus area score .61).

Regarding T/D discrimination, there was a uniform decline across the delay, in contrast to Dowling et al.
(2001) where T/D discrimination remained essentially flat. As this result pattern was the same for expressive and mechanical stimuli, this decline can be attributed to the faster tempi (i.e., shorter delays) in the present experiment, since that was the main difference with previous studies. It could be that the decreasing T/D pattern arises from better T/D performance at the short delay here, with an area score of 0.84 at $2.5 \mathrm{~s}$ (versus 0.78 at $5 \mathrm{~s}$ in the earlier studies). This difference could be the result of the potential presence of an "echoic" trace of the target at the 2-s delay, making D lures easier to reject. This benefit vanished for the longer delay here $(9.5 \mathrm{~s})$, where $\mathrm{T} / \mathrm{D}$ discrimination was at 0.78 and thus at comparable performance level to previously used longer delays, notably 0.77 at $15 \mathrm{~s}$ and 0.77 at $30 \mathrm{~s}$ (Dowling et al., 2001, Experiment 2).

It is interesting that whereas the pattern of hits and false alarms differed markedly between expressive and mechanical conditions for less-experienced participants, the more experienced participants showed similar patterns for both conditions. It seems as though the more-experienced participants brought to the task a well-practiced set of skills for encoding and remembering musical patterns, and applied them whether the stimuli were expressively played or not. The lessexperienced participants, on the other hand, were more responsive to the conditions of presentation, and (for example) took advantage of the literal repetition in the mechanical $\mathrm{T}$ items to achieve a high hit rate, and-by contrast-a lower false-alarm rate for S lures. However, at the longer delay, the hit rate for $\mathrm{T}$ items was higher for expressive than mechanical versions (while not differing in false alarms for $\mathrm{S}$ lures), suggesting that the initial difficulty for expressive material was overcome and expressive variability might have led to a benefit in target recognition.

In conclusion, we here extended the previously reported memory improvement phenomenon to realistic performances-a result suggesting the influence of slowed-down, but still continuing binding processes during the continuous ongoing listening of a musical piece.

\section{Author Note}

Correspondence concerning this article should be addressed to Barbara Tillmann, CNRS UMR5292, INSERM U1028, Centre de Recherche en Neurosciences de Lyon, Equipe Cognition Auditive et Psychoacoustique, 50 Avenue Tony Garnier, F-69366 Lyon cedex 01. E-mail: btillmann@olfac.univ-lyon1.fr 


\section{References}

Chapin, H., Jantzen, K., Scott Kelso, J. A., Steinberg, F., \& LARge, E. (2010) Dynamic emotional and neural responses to music depend on performance expression and listener experience. PLoS ONE, 5(12), e13812. doi:10.1371/ journal.pone.0013812

Clarke, E. F., \& Windsor, W. L. (2000). Real and simulated expression: A listening study. Music Perception, 17, 277-313.

Dowling, W. J., Kwak, S.-Y., \& Andrews, M. W. (1995). The time course of recognition of novel melodies. Perception and Psychophysics, 57, 197-210.

Dowling, W. J., \& Tillmann, B. (2013). Memory improvement while hearing music: Effects of structural continuity on feature binding. Manuscript in preparation.

Dowling, W. J., Tillmann, B., \& Ayers, D. (2001). Memory and the experience of hearing music. Music Perception, 19, 249-276.

Dowling, W. J., Tillmann, B., \& Magner, H. (2013). Memory improvement for popular music with wide-awake listeners. Manuscript in preparation.

Gardiner, J. M., Kaminska, Z., Dixon, M., \& Java, R. I. (1996). Repetition of previously novel melodies sometimes increases both remember and know responses in recognition memory. Psychonomic Bulletin and Review, 3, 366-371.

Kaernbach, C. (2004). The memory of noise. Experimental Psychology, 51(4), 240-248.

Koelsch, S., Kilches, S., Steinbeis, N., \& Schelinski, S. (2008). Effects of unexpected chords and of performer's expression on brain responses and electrodermal activity. PLoS ONE, 3(7), e2631. doi:10.1371/journal.pone.0002631
Koelsch, S., \& Mulder, J. (2002). Electric brain responses to inappropriate harmonies during listening to expressive music. Clinical Neurophysiology, 113, 862-869

Lerdahl, F., \& JACKendofF, R. (1983). A generative theory of tonal music. Cambridge, MA: MIT Press.

Palmer, C. (1996). Anatomy of a performance: Sources of musical expression. Music Perception, 13, 433-453.

Palmer, C., \& Krumhansl, C. L. (1987). Pitch and temporal contributions to musical phrase perception: Effects of harmony, performance timing, and familiarity. Perception and Psychophysics, 41, 505-518.

Peretz, I., Gaudreaux, D., \& Bonnel, A.-M. (1998). Exposure effects on music preference and recognition. Memory and Cognition, 26, 884-902.

Swets, J. A. (1973). The relative operating characteristic in psychology. Science, 182, 990-1000.

Tillmann, B., \& Dowling, W. J. (2007). Memory decreases for prose, but not for poetry. Memory and Cognition, 35, 628-639.

Treisman, A. M. (2006). Object tokens, binding, and visual memory. In H. D. Zimmer, A. Mecklinger, \& U. Lindenberger (Eds.), Handbook of binding and memory: Perspectives from cognitive neuroscience (pp. 315-338). Oxford, UK: Oxford University Press.

Verde, M. F., Macmillan, N. A., \& Rotello, C. M. (2006). Measures of sensitivity based on a single hit rate and false-alarm rate: The accuracy, precision, and robustness of $d$, $A_{z}$, and A'. Perception and Psychophysics, 68, 643-654.

Vieillard, S., Roy, M., \& Peretz, I. (2011). Expressiveness in musical emotions. Psychological Research, 76, 641-653. doi: 10.1007/s00426-011-0361-4 
